\title{
Estimação de Parâmetros Genéticos para Produção de Leite no Dia do Controle e Produção Acumulada até 305 dias, para as Primeiras Lactações de Vacas da Raça Caracu' Lenira El Faro 2,3 , Lucia Galvão de Albuquerque ${ }^{4,5}$
}

\begin{abstract}
RESUMO - Foram estimados parâmetros genéticos para produção de leite no dia do controle e produção acumulada até 305 dias (P305) de primeiras lactações de vacas da raça Caracu. O modelo animal considerado conteve efeito genético aditivo, como aleatório, além dos efeitos fixos de grupo contemporâneo e da idade ao parto, como covariável. Foram definidos dois grupos contemporâneos para explicar a variação ocorrida nas produções em cada controle leiteiro, compostos por ano, semana do controle e retiro (gc1) ou ano, mês do controle e retiro ( $\mathrm{gc}$ 2). Os componentes de variância foram estimados pelo método da máxima verossimilhança restrita. As variâncias fenotípicas, residuais e genéticas foram maiores no início da lactação, entretanto, a variância genética aditiva foi proporcionalmente menor em relação às demais variâncias. As estimativas de herdabilidade oscilaram entre 0,09 e 0,32 e foram maiores no final da lactação, indicando maior variabilidade genética nesse período. As correlações genéticas $\left(\mathrm{r}_{\mathrm{a}}\right)$ entre as produções em cada controle foram positivas e maiores, quanto menor a distância entre eles. A herdabilidade para P305 foi de 0,27 e as $r_{a}$ desta com os controles, positivas e elevadas, principalmente no meio da lactação. Os resultados indicam que a seleção direta para P305 proporciona maiores ganhos genéticos para essa característica que a obtida por meio de resposta correlacionada para as produções em cada controle.
\end{abstract}

Palavras-chave: bovinos leiteiros, componentes de variância, correlações, critério de seleção, produção de leite

\section{Estimation of Genetic Parameters for First Lactation Test-Day Records and Total Milk Yield for Caracu Cows}

\begin{abstract}
Genetic parameters for first lactation test-day milk yields and 305 day milk yield (M305) for Caracu cows were estimated. The test-day animal model included additive genetic effect as random and contemporary group and age of cow (quadratic regression) as fixed effects. Two contemporary groups were defined to explain variation, composed by year, week of test and paddock ( $g c 1$ ) or year, week of test and paddock (gc2). Variance components were estimated by Restricted Maximum Likelihood. Phenotypic, residual and genetic additive variances were higher at the beginning of lactation, however the genetic additive variances were proportionally smaller than the other variances. Heritability estimates ranged from 0.09 to 0.32 and were higher at the end of lactation, indicating larger genetic variability in this period. The genetic correlation $\left(r_{a}\right)$ among test-days were positive and decreased with the increase in lag between tests. Heritability for M305 was 0.27 and the $r_{a}$ between test days and M305 were positive and high, mainly for mid lactation. The results indicate greater genetic response in M305 through direct selection rather than by correlated response based on test-day milk yield selection.
\end{abstract}

Key Words: correlation, dairy cattle, heritability, milk yield, selection criterion, variance components

\section{Introdução}

A produção de leite no dia do controle vem sendo proposta como um critério de seleção alternativo à produção acumulada em até 305 dias, por meio de metodologia denominada "test-day model" (TDM). O TDM é um procedimento estatístico em que as produções no dia do controle são analisadas como características distintas, consideradas em análises unicaracterísticas ou multicaracterísticas. O TDM mais simples é o modelo de repetibilidade, que assu- me estrutura unicaracterística, variâncias homogêneas entre todos os controles e correlações genéticas entre eles iguais à unidade, o que não ocorre na realidade. Tem-se observado que as correlações genéticas entre os controles são elevadas, principalmente entre os adjacentes, mas não iguais a um.

$\mathrm{O}$ uso de TDM possibilita que um animal seja avaliado com apenas um controle, diminuindo assim os custos com controle leiteiro, além de acelerar o processo de avaliação genética, uma vez que não seria preciso esperar o término da lactação para

\footnotetext{
1 Parte da tese de doutorado do primeiro autor, financiada pelo CNPq

${ }^{2}$ Aluno de doutorado - FCAV/UNESP, Rod. Paulo D. Castelani km 8, CEP 14870-000, Jaboticabal-SP

3 Pesquisador do Instituto de Zootecnia, SP. E.mail: lenira.ddd@apta.sp.gov.br

${ }^{4}$ Prof. assitente doutor - FCAV/UNESP, Jaboticabal-SP. E.mail: Igalb@fcav.unesp.br

${ }^{5}$ Pesquisador do CNPq.
} 
incluir as informações no conjunto de dados. Outras vantagens estariam relacionadas ao melhor detalhamento na modelagem de efeitos sistemáticos que influenciam a produção de leite em períodos específicos da lactação, impossíveis de serem considerados quando a produção total acumulada é usada. Além disso, seria desnecessário o uso de fatores de extensão para lactações em andamento. De acordo com alguns autores, quando a produção total de leite é estimada por meio de fatores de extensão, eliminase a existência de variabilidade na forma das curvas de lactação de cada animal, tendo como conseqüência a eliminação de alguma variação genética para a produção. Por outro lado, a exclusão de lactações curtas ou incompletas na avaliação genética pode causar vícios devido à seleção de dados. O TDM proporcionaria, portanto, menores vícios na estimação dos componentes de variância e, conseqüentemente, nos valores genéticos preditos dos animais (Ptak \& Schaeffer, 1993; Swalve, 1995a,b; Gadini, 1997; Swalve, 1998). As $h^{2}$ estimadas para produção no dia do controle, em vacas Holandesas, têm se apresentado próximas ou superiores às encontradas para produção acumulada, sendo o meio da lactação, em torno do quarto ou quinto controle, mais herdável que o início e o final (Swalve, 1995a,b; Kettunen et al., 1998; Machado et al., 1998).

O objetivo do presente estudo foi estimar parâmetros genéticos para produção de leite no dia do controle e para a produção de leite até 305 dias, procurando, assim, detectar períodos em que ocorreu maior variabilidade genética e apontar possíveis critérios de seleção alternativos para o rebanho estudado.

\section{Material e Métodos}

No presente estudo foram utilizadas 2155 primeiras lactações de vacas da raça Caracu, iniciadas entre os anos de 1978 a 1998, pertencentes à Fazenda Chiqueirão, Poços de Caldas, MG. Os controles foram registrados semanalmente, provenientes de ordenha manual, realizada duas vezes ao dia, com a presença do bezerro. Os animais foram alimentados basicamente a pasto, nativo ou cultivado, com suplementação no período das secas. A fazenda, quanto aos locais de ordenha, é dividida em 15 retiros, sendo dois deles maternidade. Durante a lactação, o animal passou por até três retiros, dependendo da fase da lactação e do nível de produção.

A lactação foi truncada aos 305 dias e dividida em

Tabela 1 - Médias, desvios-padrão e coeficientes de variação (CV\%) para a produção de leite, de acordo com as classes de controles leiteiros

Table 1 - Means, standard deviations and coefficients of variation for milk yield, according to the classes of day in milk

\begin{tabular}{|c|c|c|c|c|c|c|}
\hline $\begin{array}{l}\text { Classe de } \\
\text { controle } \\
\text { Class of day } \\
\text { inmilk }\end{array}$ & $\begin{array}{c}\text { Semana de } \\
\text { lactação } \\
\text { Week }\end{array}$ & $\begin{array}{c}\text { Dias em } \\
\text { lactação } \\
\text { Days in milk }\end{array}$ & $\begin{array}{c}\mathrm{N}^{\mathrm{o}} \cdot \mathrm{de} \\
\text { observações } \\
\text { N. of records }\end{array}$ & $\begin{array}{c}\text { Média }(\mathrm{kg}) \\
\text { Mean }(\mathrm{kg})\end{array}$ & $\begin{array}{c}\text { Desvio-padrão }(\mathrm{kg}) \\
\text { Standard deviation }(\mathrm{kg})\end{array}$ & $\mathrm{CV}(\%)$ \\
\hline 1 & 2 & $12-18$ & 2059 & 7,25 & 2,53 & 35 \\
\hline 2 & 4 & 26- 32 & 2076 & 7,42 & 2,51 & 34 \\
\hline 3 & 6 & $40-46$ & 2087 & 7,18 & 2,34 & 33 \\
\hline 4 & 8 & 54- 60 & 2092 & 6,99 & 2,09 & 30 \\
\hline 5 & 10 & 68- 74 & 2120 & 6,68 & 1,98 & 30 \\
\hline 6 & 12 & 82- 88 & 2129 & 6,30 & 1,89 & 30 \\
\hline 7 & 14 & $96-102$ & 2130 & 5,80 & 1,77 & 30 \\
\hline 8 & 16 & $110-116$ & 2138 & 5,42 & 1,69 & 31 \\
\hline 9 & 18 & $124-130$ & 2142 & 5,09 & 1,63 & 32 \\
\hline 10 & 20 & $138-144$ & 2132 & 4,93 & 1,60 & 32 \\
\hline 11 & 22 & $152-158$ & 2130 & 4,76 & 1,59 & 33 \\
\hline 12 & 24 & $166-172$ & 2137 & 4,60 & 1,56 & 34 \\
\hline 13 & 26 & $180-186$ & 2135 & 4,46 & 1,55 & 35 \\
\hline 14 & 28 & $194-200$ & 2134 & 4,32 & 1,55 & 36 \\
\hline 15 & 30 & $208-214$ & 2136 & 4,15 & 1,57 & 38 \\
\hline 16 & 32 & $222-228$ & 2121 & 3,97 & 1,59 & 40 \\
\hline 17 & 34 & $236-242$ & 2099 & 3,69 & 1,61 & 44 \\
\hline 18 & 36 & $250-256$ & 2026 & 3,41 & 1,60 & 47 \\
\hline 19 & 38 & $264-270$ & 1875 & 3,16 & 1,56 & 49 \\
\hline 20 & 40 & $278-284$ & 1614 & 2,96 & 1,49 & 50 \\
\hline 21 & 42 & $299-305$ & 1235 & 2,76 & 1,40 & 51 \\
\hline
\end{tabular}


43 períodos semanais, uma vez que o sistema de controle leiteiro adotado pela propriedade foi semanal. Nas análises foram considerados controles quinzenais, iniciando a partir da $2^{\mathrm{a}}$ semana, totalizando assim 21 classes de controles leiteiros (Tabela 1).

As produções acumuladas até 305 dias (P305) foram calculadas com base no método escada corrigido, de acordo com Bianchini Sobrinho (1986):

$$
P 305=Y_{1} X_{1}+\sum_{i=2}^{n} y_{i} x_{i}+Y_{n} X_{n}
$$

em que: $\mathrm{Y}_{1}=$ produção diária de leite no primeiro controle; $X_{1}=$ amplitude do intervalo do início da lactação ao primeiro controle; $\mathrm{n}=$ número de controles; $y_{i}=$ produção diária de leite no i-ésimo controle; $\mathrm{x}_{\mathrm{i}}=$ amplitude do intervalo de controles; $\mathrm{Y}_{\mathrm{n}}=$ produção diária de leite no último controle; $\mathrm{X}_{\mathrm{n}}=$ amplitude do intervalo do último controle ao final da lactação.

As características foram analisadas por meio de modelo animal, uni e bicaracterístico, sendo que as análises bicaracterísticas foram realizadas entre as 21 produções de leite no dia do controle e entre estas e a produção acumulada até 305 dias.

Os modelos matemáticos incluíram efeito genético aditivo direto, como aleatório, e os efeitos fixos de grupo contemporâneo e idade da vaca ao parto, regressão linear e quadrática, como covariável. $\mathrm{O}$ grupo contemporâneo, para a P305, foi composto pelas variáveis ano e mês do parto, em um total de 179 classes. Para os controles individuais foram definidos dois grupos contemporâneos, compostos pelas variáveis ano, semana de realização do controle e retiro (gc1) ou ano, mês de realização do controle e retiro (gc2). Para as análises bicaracterísticas, utilizou-se apenas o gc2, uma vez que havia pequeno número de observações em cada grupo, o que gerou problemas de convergência. Lactações contendo menos que seis controles semanais foram desconsideradas nas análises. Os modelos podem ser representados em sua forma matricial por:

$$
\mathbf{y}=\mathbf{X b}+\mathbf{Z a}+\mathbf{e}
$$

em que y é o vetor da produção de leite acumulada até 305 dias ou em cada controle leiteiro; b, o vetor de efeitos fixos contendo grupo contemporâneo e a covariável idade ao parto; a, o vetor de soluções para os efeitos aleatórios genético aditivos; $\mathrm{XeZ}$, as matrizes de incidência para os efeitos fixos e genético aditivo, respectivamente; e, o vetor de resíduos aleatórios.
As pressuposições em relação às médias e variâncias para as análises bicaracterísticas são:

$$
E\left[\begin{array}{l}
\mathbf{y} \\
\mathbf{a} \\
\mathbf{e}
\end{array}\right]=\left[\begin{array}{c}
\mathbf{X b} \\
\mathbf{0} \\
\mathbf{0}
\end{array}\right] \quad \text { e } \quad V\left[\begin{array}{l}
\mathbf{a} \\
\mathbf{e}
\end{array}\right]=\left[\begin{array}{cc}
\mathbf{G} & \mathbf{0} \\
\mathbf{0} & \mathbf{R}
\end{array}\right]
$$

sendo que: $\mathbf{G}=\mathbf{A} \otimes \mathbf{G}_{\mathbf{0}}$ é a matriz de (co)variâncias genéticas aditivas entre as características e

$$
\mathbf{G}_{\mathbf{0}}=\left(\begin{array}{cc}
\sigma_{a_{i}}^{2} & \sigma_{a_{i} a_{j}} \\
& \sigma_{a_{j}}^{2}
\end{array}\right)
$$

$\mathrm{A}=$ matriz de parentesco entre os animais; $\otimes=$ produto de Kroenecker entre matrizes; $\boldsymbol{\sigma}^{\mathbf{2}}{ }_{a i}$ e $\boldsymbol{\sigma}^{\mathbf{2}}{ }_{a j}=$ variâncias genéticas aditivas; $\boldsymbol{\sigma}_{a i a j}=$ covariância genética entre as características i e $\mathrm{j} ; \mathrm{R}=\mathrm{I} \otimes \mathrm{R}_{0}$, é a matriz de (co)variâncias residuais entre as características e

$$
\mathbf{R}_{\mathbf{0}}=\left(\begin{array}{cc}
\sigma_{e_{i}}^{2} & \sigma_{e_{i} e_{j}} \\
& \sigma_{e_{j}}^{2}
\end{array}\right)
$$

$\mathrm{I}=$ matriz identidade; $\sigma_{e i}^{2}$ e $\sigma^{2}{ }_{e j}=$ variâncias residuais; $\sigma_{e i e j}=$ covariância residual entre as características i e j.

Para as análises unicaracterísticas, as pressuposições são basicamente as mesmas, exceto que $\mathrm{G}=\mathrm{A}$ $\sigma_{a}^{2}$ e $\mathrm{R}=\mathrm{I} \sigma_{e}^{2}$.

Os componentes de variância foram estimados pelo método da Máxima Verossimilhança Restrita, que utiliza algoritmo livre de derivadas, disponível no pacote MTDFREML (Boldman et al., 1993).

Os ganhos genéticos e as respostas correlacionadas foram calculados para todas as características estudadas, considerando-se uma intensidade de seleção constante. As fórmulas usadas podem ser representadas por:

$$
\begin{gathered}
\Delta G=i \sigma_{Y} h^{2}, \\
R C_{2.1}=r_{a 12} h_{y 1} h_{y 2} i \sigma_{y 2},
\end{gathered}
$$

em que: $\Delta \mathrm{G}=$ ganho genético mediante seleção direta para a característica; $\mathrm{RC}_{2.1}=$ resposta correlacionada 
para a característica 2, mediante seleção direta para a característica $1 ; \sigma_{\mathrm{y}}=$ desvio-padrão fenotípico da característica a ser selecionada; $\mathrm{i}=$ intensidade de seleção; $\mathrm{r}_{\mathrm{a} 12}$ = correlação genética entre as características $1 \mathrm{e} 2$.

\section{Resultados e Discussão}

As produções de leite em cada controle apresentaram altos CV\% (Tabela 1), indicando que há grande variação na forma da curva de lactação dos animais desse rebanho. A maior variação nos controles do final da lactação era esperada, uma vez que o número de observações foi menor no final da lactação, período próximo à secagem das vacas.

Como esperado, para gc1 houve maior número de grupos contemporâneos (Tabela 2), variando de 544 a 781 grupos para as 21 características, e baixo número de observações em cada subclasse. Do total de gc1, 39,2\% deles continham apenas uma observação e $61,5 \%$ deles continham até duas observações. Para gc2 (Tabela 3), o número de grupos variou de 258 a 390 , sendo que a porcentagem de gc2 com apenas uma observação foi de $20,1 \%$ e com até duas observações caiu para $33,6 \%$.

A presença de grupos contendo poucas informações era esperada nesse estudo, por haver um número pequeno de primeiras lactações, dispersas em vários anos. Além disso, evitou-se eliminar grupos com poucas informações, procurando não alterar a estrutura da lactação propriamente dita. O problema de haver muitos grupos contemporâneos contendo poucas observações por grupo foi observado em outros estudos. Pander et al. (1992) usaram as variáveis rebanho, ano e mês do controle (RAM), como base dos grupos contemporâneos, em vez de rebanho e data do controle (RDC), e, mesmo assim, 56\% dos níveis de RAM apresentaram apenas uma observação. Gadini (1997), que utilizou a base de dados do Mid States Dairy Processing Center, EUA, com 364.000 primeiras lactações de vacas Holandesas, observou 53\% de grupos contemporâneos (RDC) contendo apenas uma observação.

Resultados provenientes da literatura têm demonstrado que o maior detalhamento dos fatores ambientais que afetam a produção em períodos espe-

Tabela 2 - Estimativas de variâncias genéticas $\left(\sigma_{\mathrm{a}}^{2}\right)$, residuais $\left(\sigma_{\mathrm{e}}^{2}\right)$, fenotípicas $\left(\sigma_{\mathrm{p}}^{2}\right)$ e herdabilidades $\left(\mathrm{h}^{2}\right)$ para as 21 classes de controles leiteiros, para a definição de gc1

Table 2 - Estimates of genetic $\left(\sigma_{a}^{2}\right)$, residual $\left(\sigma_{e}^{2}\right)$ and phenotypic $\left(\sigma_{p}^{2}\right)$ variances and heritability $\left(h^{2}\right)$ for the 21 classes of days in milk, by gc1

\begin{tabular}{lccccccc}
\hline $\begin{array}{l}\text { Classe de controle } \\
\text { Class of day in milk }\end{array}$ & $\begin{array}{c}\text { Semana de lactação } \\
\text { Week }\end{array}$ & $\begin{array}{c}\text { Dias em lactação } \\
\text { Days in milk }\end{array}$ & $\begin{array}{c}\text { No. de GC } \\
\text { No. of CG }\end{array}$ & $\sigma^{2}{ }_{\mathrm{a}}$ & $\sigma^{2}{ }_{\mathrm{e}}$ & $\sigma^{2} \mathrm{p}$ & $\mathrm{h}^{2}$ \\
\hline 1 & 2 & $12-18$ & 709 & 1,048 & 3,262 & 4,310 & 0,24 \\
2 & 4 & $26-32$ & 755 & 0,722 & 2,941 & 3,663 & 0,20 \\
3 & 6 & $40-46$ & 765 & 0,487 & 2,419 & 2,906 & 0,17 \\
4 & 8 & $54-60$ & 770 & 0,516 & 2,119 & 2,635 & 0,20 \\
5 & 10 & $68-74$ & 780 & 0,363 & 1,918 & 2,280 & 0,16 \\
6 & 12 & $82-88$ & 777 & 0,319 & 1,762 & 2,081 & 0,15 \\
7 & 14 & $96-102$ & 779 & 0,294 & 1,475 & 1,769 & 0,17 \\
8 & 16 & $110-116$ & 780 & 0,429 & 1,377 & 1,806 & 0,24 \\
9 & 18 & $124-130$ & 781 & 0,331 & 1,250 & 1,581 & 0,21 \\
10 & 20 & $138-144$ & 781 & 0,264 & 1,327 & 1,591 & 0,17 \\
11 & 22 & $152-158$ & 780 & 0,492 & 1,131 & 1,623 & 0,30 \\
12 & 24 & $166-172$ & 779 & 0,332 & 1,188 & 1,520 & 0,22 \\
13 & 26 & $180-186$ & 779 & 0,335 & 1,222 & 1,556 & 0,22 \\
14 & 28 & $194-200$ & 777 & 0,326 & 1,192 & 1,519 & 0,21 \\
15 & 30 & $208-214$ & 779 & 0,365 & 1,147 & 1,511 & 0,24 \\
16 & 32 & $222-228$ & 779 & 0,227 & 1,228 & 1,455 & 0,16 \\
17 & 34 & $236-242$ & 778 & 0,277 & 1,358 & 1,635 & 0,17 \\
18 & 36 & $250-256$ & 773 & 0,173 & 1,387 & 1,560 & 0,11 \\
19 & 38 & $264-270$ & 726 & 0,152 & 1,331 & 1,489 & 0,11 \\
20 & 40 & $278-284$ & 662 & 0,302 & 1,303 & 1,605 & 0,19 \\
21 & 42 & $299-305$ & 544 & 0,426 & 0,848 & 1,274 & 0,33 \\
\hline
\end{tabular}


cíficos da lactação poderiam contribuir para diminuição das variações devido ao ambiente temporário. Meyer et al. (1989) obtiveram menores variâncias residuais, quando especificaram o grupo contemporâneo por meio das variáveis rebanho-data do controle, em comparação com rebanho-ano-estação. O mesmo foi observado por Swalve (1995) e Gadini (1997). No presente estudo, observou-se redução na variância ambiental, quando se utilizou ano-semana do controle-retiro (gc1) em relação à ano-mês do controleretiro ( $g c 2)$, com menores variâncias fenotípicas, na maioria dos controles, para gc1. Isso resultou em maiores $\sigma_{a}^{2}$, proporcionalmente, para gc1 que para gc2, e maiores $h^{2}$ em alguns períodos da lactação (Tabelas 2 e 3 ).

As $\sigma_{p}^{2}$ para gc1 (Figura 1) foram elevadas no início da lactação, decrescendo no decorrer do período e estabilizando-se por volta das 18 semanas de lactação até o final. A mesma tendência foi observada para as variâncias residuais. As variâncias genéticas foram bem menores que as residuais e mantiveram-se sem grandes oscilações por todo o período da lactação, aumentando levemente no início e no final.
Houve maior distância das variâncias genéticas em relação às fenotípicas no início da lactação, se comparadas ao final da lactação, e, conseqüentemente, os valores de $\mathrm{h}^{2}$ foram maiores no final da lactação. As variâncias mostraram tendências semelhantes para gc2, exceto que as $\sigma_{2}{ }^{\mathrm{e}}$ foram pouco maiores. As estimativas de $h^{2}$ apresentaram valores entre 0,09 ( $12^{\mathrm{a}}$ semana) e 0,32 ( $42^{\mathrm{a}}$ semana), para gc2. Os valores oscilaram no decorrer da lactação, sendo que o início foi menos herdável, no geral, que o meio e o final. A segunda metade da lactação, a partir da $22^{\mathrm{a}}$ semana, apresentou $\mathrm{h}^{2}$ mais elevadas, sendo que as maiores estimativas foram obtidas para as $28^{\mathrm{a}}, 32^{\mathrm{a}}$, $40^{\mathrm{a}}$ e $42^{\mathrm{a}}$ semanas. Para gc1, as $\mathrm{h}^{2}$ foram maiores, variando entre 0,11 ( $36^{\mathrm{a}}$ e $38^{\mathrm{a}}$ semanas) e 0,33 (42 semana). A tendência para gc1 diferiu um pouco da observada para gc2, com $\mathrm{h}^{2}$ maiores da $14^{\mathrm{a}}$ até a $30^{\mathrm{a}}$ semana. No final da lactação, $40^{\mathrm{a}}$ e $42^{\mathrm{a}}$ semanas, as estimativas foram maiores que nos demais períodos para as duas definições de grupo contemporâneo.

Os resultados desse estudo discordam dos encontrados na literatura, para gado Holandês, principalmente para o final da lactação (Meyer et al., 1989;

Tabela 3 - Estimativas de variâncias genéticas $\left(\sigma^{2}\right.$ ), residuais $\left(\sigma^{2} e\right)$, fenotípicas $\left(\sigma^{2} p\right)$ e herdabilidades $\left(h^{2}\right.$ uni e $h^{2}$ bi) para as 21 classes de controles leiteiros, para a definição de gc2

Table 3 - Estimates of genetic $\left(\sigma_{a}^{2}\right)$, residual $\left(\sigma_{e}^{2}\right)$ and phenotypic $\left(\sigma_{p}^{2}\right)$ variances and heritability $\left(h^{2}\right.$ uniand $h^{2}$ bi) for the 21 classes of days in milk, by gc2

\begin{tabular}{lcccccccc}
\hline $\begin{array}{l}\text { Classe de controle } \\
\text { Class of day in milk }\end{array}$ & $\begin{array}{c}\text { Semana de lactação } \\
\text { Week }\end{array}$ & $\begin{array}{c}\text { Dias em lactação } \\
\text { Days in milk }\end{array}$ & $\begin{array}{c}\text { No. de GC } \\
\text { N. of CG }\end{array}$ & $\sigma^{2}{ }_{\mathrm{a}}$ & $\sigma_{\mathrm{e}}^{2}$ & $\sigma_{\mathrm{p}}^{2}$ & $\mathrm{~h}^{2} \mathrm{uni}^{2} \mathrm{~h}^{2} \mathrm{bi}^{2}$ \\
\hline 1 & 2 & $12-18$ & 305 & 0,935 & 3,512 & 4,447 & 0,21 & 0,22 \\
2 & 4 & $26-32$ & 386 & 0,582 & 3,181 & 3,763 & 0,15 & 0,15 \\
3 & 6 & $40-46$ & 390 & 0,472 & 2,586 & 3,057 & 0,15 & 0,16 \\
4 & 8 & $54-60$ & 374 & 0,319 & 2,259 & 2,579 & 0,12 & 0,13 \\
5 & 10 & $68-74$ & 370 & 0,318 & 2,011 & 2,329 & 0,14 & 0,14 \\
6 & 12 & $82-88$ & 374 & 0,182 & 1,907 & 2,089 & 0,09 & 0,09 \\
7 & 14 & $96-102$ & 363 & 0,175 & 1,664 & 1,838 & 0,10 & 0,11 \\
8 & 16 & $110-116$ & 380 & 0,240 & 1,536 & 1,776 & 0,14 & 0,14 \\
9 & 18 & $124-130$ & 366 & 0,243 & 1,338 & 1,581 & 0,15 & 0,15 \\
10 & 20 & $138-144$ & 366 & 0,246 & 1,333 & 1,579 & 0,16 & 0,15 \\
11 & 22 & $152-158$ & 361 & 0,297 & 1,305 & 1,602 & 0,19 & 0,18 \\
12 & 24 & $166-172$ & 375 & 0,232 & 1,303 & 1,534 & 0,15 & 0,15 \\
13 & 26 & $180-186$ & 367 & 0,211 & 1,275 & 1,487 & 0,14 & 0,14 \\
14 & 28 & $194-200$ & 370 & 0,364 & 1,136 & 1,500 & 0,24 & 0,23 \\
15 & 30 & $208-214$ & 371 & 0,219 & 1,307 & 1,526 & 0,14 & 0,15 \\
16 & 32 & $222-228$ & 366 & 0,329 & 1,188 & 1,518 & 0,22 & 0,22 \\
17 & 34 & $236-242$ & 377 & 0,268 & 1,394 & 1,662 & 0,16 & 0,16 \\
18 & 36 & $250-256$ & 357 & 0,201 & 1,397 & 1,598 & 0,13 & 0,14 \\
19 & 38 & $264-270$ & 355 & 0,213 & 1,350 & 1,563 & 0,14 & 0,15 \\
20 & 40 & $278-284$ & 314 & 0,362 & 1,246 & 1,608 & 0,22 & 0,25 \\
21 & 42 & $299-305$ & 258 & 0,454 & 0,977 & 1,432 & 0,32 & 0,31 \\
\hline
\end{tabular}

1. análises unicaracterísticas; 2. médias estimadas das análises bicaracterísticas.

1. unitrait analysis; 2. Estimated averages from bitrait analysis. 

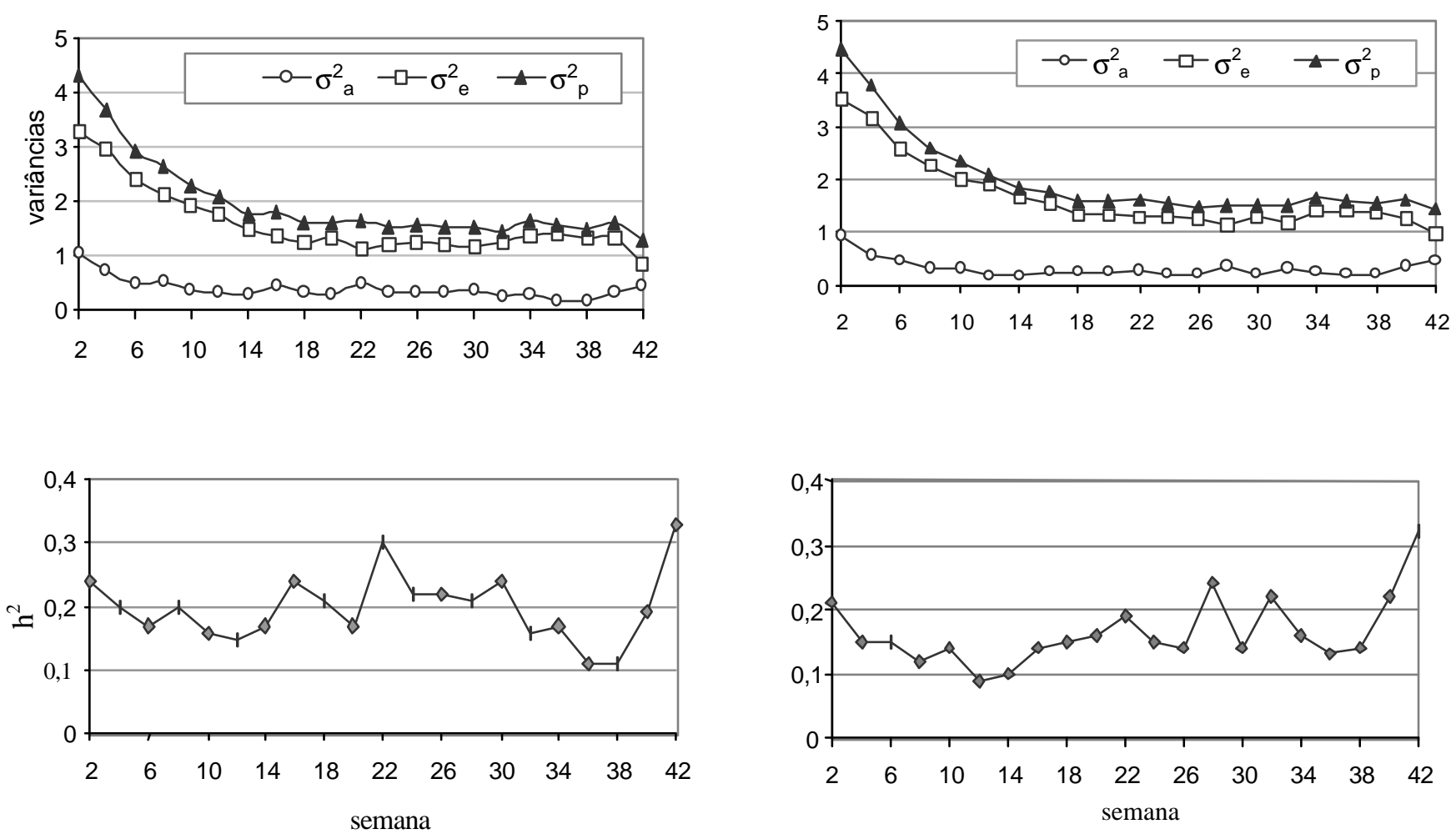

Figura 1 - Estimativas de variâncias fenotípicas $\left(\sigma_{p}^{2}\right)$, genéticas aditivas $\left(\sigma_{a}^{2}\right)$ e residuais $\left(s^{2}\right)$ para produções de leite no dia do controle, usando gc1 (acima, à esquerda) e gc2 (acima, à direita) e, estimativas de $\mathrm{h}^{2}$, usando gc1 (abaixo, à esquerda) e gc2 (abaixo, à direita).

Figure 1 - Estimates of phenotypic, additive genetic and residual variances for test day milk yield, using gc1 (up, left) and gc2 (up, right) and heritability using gc1 (down, left) and gc2 (down, right).

Kettunen et al., 1998; Machado et al., 1998). Os valores descritos na literatura têm indicado, em geral, que o meio da lactação é mais herdável que as demais fases. Meyer et al. (1989) estimaram maiores $h^{2}$ no segundo trimestre da lactação. A mesma tendência foi observada por Pander et al. (1992). Swalve (1995b) obteve as maiores $\mathrm{h}^{2}$ entre o $4^{\circ}$ e o $8^{\circ}$ controles, sendo igual a 0,43 no $8^{\circ}$ controle. Olori (1997), para produções semanais de vacas Holandesas, estimou as maiores $\mathrm{h}^{2}$ no final da lactação, em torno da $33^{\mathrm{a}}$ até a $42^{\mathrm{a}}$ semana. Os valores estimados por esse autor foram maiores que os do presente estudo e variaram entre 0,26 ( $2^{\mathrm{a}}$ semana) e 0,48 ( $33^{\mathrm{a}}$ semana).

Vários autores têm enfatizado que o início da lactação deveria ser desconsiderado em decisões de seleção, uma vez que grande parte da variação total não é explicada pelos modelos nesse período (Wilmink, 1987; Pander et al., 1992; Swalve, 1995a, b). Isso pode ser constatado na Figura 1, uma vez que há grande distância entre as variâncias fenotípicas e residuais e as variâncias genéticas logo no início da lactação.

Os resultados obtidos no presente estudo podem ser explicados pela tendência das lactações desse rebanho serem curtas ou terem quedas bruscas nas produções no final da lactação e, também, pela definição de grupo contemporâneo. O ideal, de acordo com a literatura, seria considerar a data do controle leiteiro, além do retiro, para definir os grupos contemporâneos, o que foi impossível, devido ao número de informações dispersas em vários anos de parto, o que gerava grande número de subclasses com baixo número de informações.

As análises bicaracterísticas entre os controles leiteiros foram realizadas apenas para gc 2 e as médias das estimativas de $\mathrm{h}^{2}$ foram muito próximas às das análises unicaracterísticas (Tabela 3 ).

As covariâncias fenotípicas, genéticas e ambientais provenientes das análises bicaracterísticas 
são apresentadas na Figura 2. As covariâncias fenotípicas, assim como as variâncias, foram maiores no início da lactação e entre controles adjacentes, diminuindo em magnitude quanto maior a distância entre os mesmos. As superfícies representando as covariâncias fenotípicas não apresentaram grandes oscilações e a maior parte das covariâncias esteve entre 0,5 e 1,5 , sendo que todos os valores foram positivos, como esperado. As covariâncias genéticas apresentaram maiores oscilações que as covariâncias fenotípicas, ocorrendo valores mais elevados no início da lactação, com queda brusca nos demais períodos. As covariâncias residuais apresentaram tendências semelhantes à superfície apresentada pelas covariâncias fenotípicas. Eram esperadas estimativas que proporcionassem superfícies mais suaves para os efeitos estudados, de acordo com o observado na literatura (Gadini, 1997).
As $r_{a}$ (Figura 3) variaram entre 0,3 e 1,0. A maioria das $r_{a}$ foi igual ou muito próxima da unidade, principalmente entre os controles consecutivos. Como a freqüência dos controles foi quinzenal, era esperado que tal fato ocorresse. Assim como a superfície apresentada para as (co)variâncias genéticas, para as $r_{a}$ ocorreram muitas oscilações, sendo que, muitas vezes, controles próximos apresentaram menores $r_{a}$ que controles mais distantes, provocando "pontas" no gráfico. As $r_{p}$ foram menores que as $r_{a}$. Os gráficos de superfícies representados pelas $r_{p}$ e $r_{e}$ foram muito parecidos em tendência e relativamente suaves quando comparados ao das $r_{a}$. A maior parte dos valores para $r_{p}$ e $r_{e}$ encontra-se entre 0,2 e 0,6 , com maiores valores entre controles mais próximos, diminuindo quando os controles se distanciam. Nota-se, de acordo com a Tabela 4, que as $r_{a}$ estimadas entre controles do início e do final da lactação foram positivas, mas

Tabela 4 - Estimativas de $h^{2}$ (diagonal), $r_{a}$ (abaixo) e $r_{p}$ (acima) entre as produções de leite em diferentes estágios da lactação

Table 4 - Estimates of $h^{2}$ (diagonal), $r_{a}$ (down) and $r_{p}$ (up) among test day milk yield in different stage of lactation

\begin{tabular}{|c|c|c|c|c|c|c|}
\hline \multicolumn{2}{|l|}{$\begin{array}{l}\text { Semana } \\
\text { Week }\end{array}$} & 2 & 4 & 6 & 8 & 10 \\
\hline $\begin{array}{l}\text { Início da } \\
\text { lactação }\end{array}$ & $\begin{array}{c}2 \\
4 \\
6 \\
8 \\
10\end{array}$ & $\begin{array}{l}\mathbf{0 , 2 1} \\
0,84 \\
0,89 \\
1,00 \\
0,71\end{array}$ & $\begin{array}{l}0,55 \\
\mathbf{0 , 1 5} \\
0,96 \\
1,00 \\
0,91\end{array}$ & $\begin{array}{r}0,46 \\
0,58 \\
\mathbf{0 , 1 5} \\
1,00 \\
0,98\end{array}$ & $\begin{array}{r}0,44 \\
0,48 \\
0,59 \\
\mathbf{0 , 1 2} \\
1,00\end{array}$ & $\begin{array}{l}0,37 \\
0,44 \\
0,49 \\
0,57 \\
\mathbf{0 , 1 4}\end{array}$ \\
\hline Semana & & 20 & 22 & 24 & 26 & 28 \\
\hline $\begin{array}{l}\text { Meio da } \\
\text { Lactação }\end{array}$ & $\begin{array}{l}20 \\
22 \\
24 \\
26 \\
28\end{array}$ & $\begin{array}{l}\mathbf{0 , 1 6} \\
1,00 \\
0,87 \\
0,93 \\
0,88\end{array}$ & $\begin{array}{l}0,59 \\
\mathbf{0 , 1 9} \\
0,89 \\
0,86 \\
0,93\end{array}$ & $\begin{array}{r}0,57 \\
0,63 \\
\mathbf{0 , 1 5} \\
0,96 \\
0,86\end{array}$ & $\begin{array}{r}0,56 \\
0,56 \\
0,60 \\
\mathbf{0 , 1 4} \\
0,86\end{array}$ & $\begin{array}{l}0,54 \\
0,55 \\
0,58 \\
0,64 \\
\mathbf{0 , 2 4}\end{array}$ \\
\hline Semana & & 32 & 34 & 36 & 38 & 40 \\
\hline $\begin{array}{l}\text { Final da } \\
\text { lactação }\end{array}$ & $\begin{array}{l}32 \\
34 \\
36 \\
38 \\
40\end{array}$ & $\begin{array}{l}\mathbf{0 , 2 2} \\
0,98 \\
0,97 \\
0,83 \\
0,90\end{array}$ & $\begin{array}{l}0,64 \\
\mathbf{0 , 1 6} \\
1,00 \\
1,00 \\
0,89\end{array}$ & $\begin{array}{r}0,60 \\
0,67 \\
\mathbf{0 , 1 3} \\
0,95 \\
1,00\end{array}$ & $\begin{array}{r}0,58 \\
0,62 \\
0,67 \\
\mathbf{0 , 1 4} \\
0,95\end{array}$ & $\begin{array}{l}0,52 \\
0,58 \\
0,61 \\
0,66 \\
\mathbf{0 , 2 2}\end{array}$ \\
\hline Semana & & 4 & 10 & 20 & 30 & 40 \\
\hline $\begin{array}{l}\text { Todos os } \\
\text { estágios }\end{array}$ & $\begin{array}{c}4 \\
10 \\
20 \\
30 \\
40\end{array}$ & $\begin{array}{l}\mathbf{0 , 1 5} \\
0,91 \\
0,93 \\
0,38 \\
0,36 \\
\end{array}$ & $\begin{array}{l}0,44 \\
\mathbf{0 , 1 4} \\
1,00 \\
0,67 \\
0,44 \\
\end{array}$ & $\begin{array}{r}0,32 \\
0,44 \\
\mathbf{0 , 1 6} \\
0,72 \\
0,85 \\
\end{array}$ & $\begin{array}{r}0,31 \\
0,39 \\
0,48 \\
\mathbf{0 , 1 4} \\
0,98 \\
\end{array}$ & $\begin{array}{r}0,25 \\
0,32 \\
0,39 \\
0,49 \\
\mathbf{0 , 2 2} \\
\end{array}$ \\
\hline
\end{tabular}



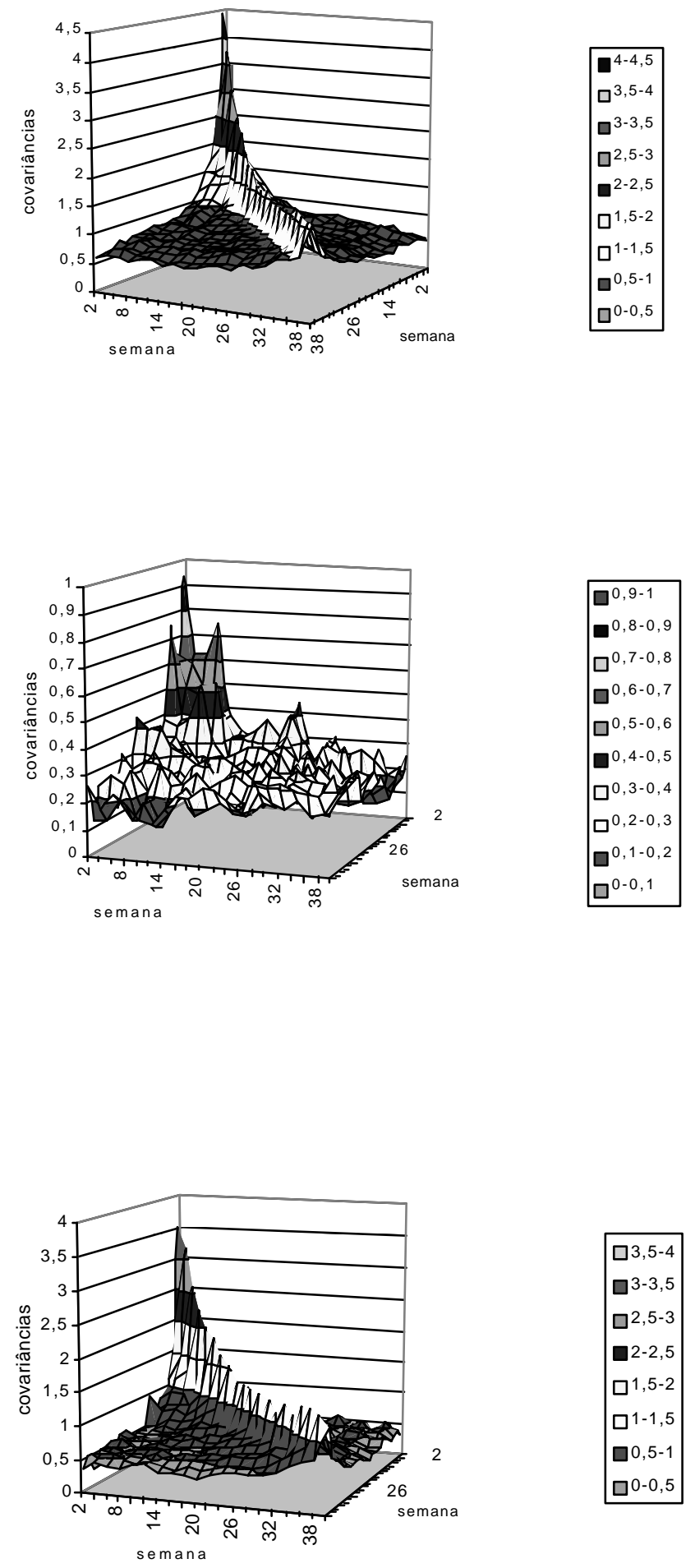

\begin{tabular}{|l|}
\hline$\square^{3,5-4}$ \\
$\square^{3-3,5}$ \\
$\square^{2,5-3}$ \\
$\square^{2-2,5}$ \\
$\square^{1,5-2}$ \\
$\square^{1-1,5}$ \\
$\square 0,5-1$ \\
$\square^{0-0,5}$ \\
\hline
\end{tabular}

Figura 2 - (Co)variâncias fenotípicas (acima), genéticas (meio) e residuais (abaixo) estimadas entre as produções de leite no dia do controle.

Figure 2 - Phenotypic, genetic and residual (co)variances estimated among test day milk yield.

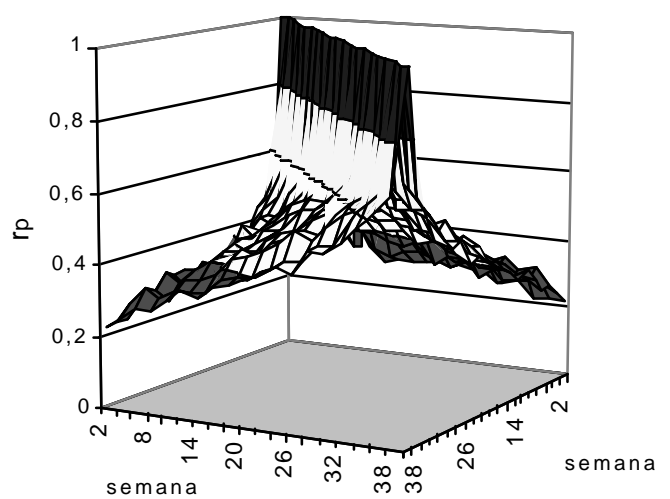

$\square^{0,8-1}$
$\square^{0,6-0,8}$
$\square^{0,4-0,6}$
$\square^{0,2-0,4}$
$\square^{0-0,2}$
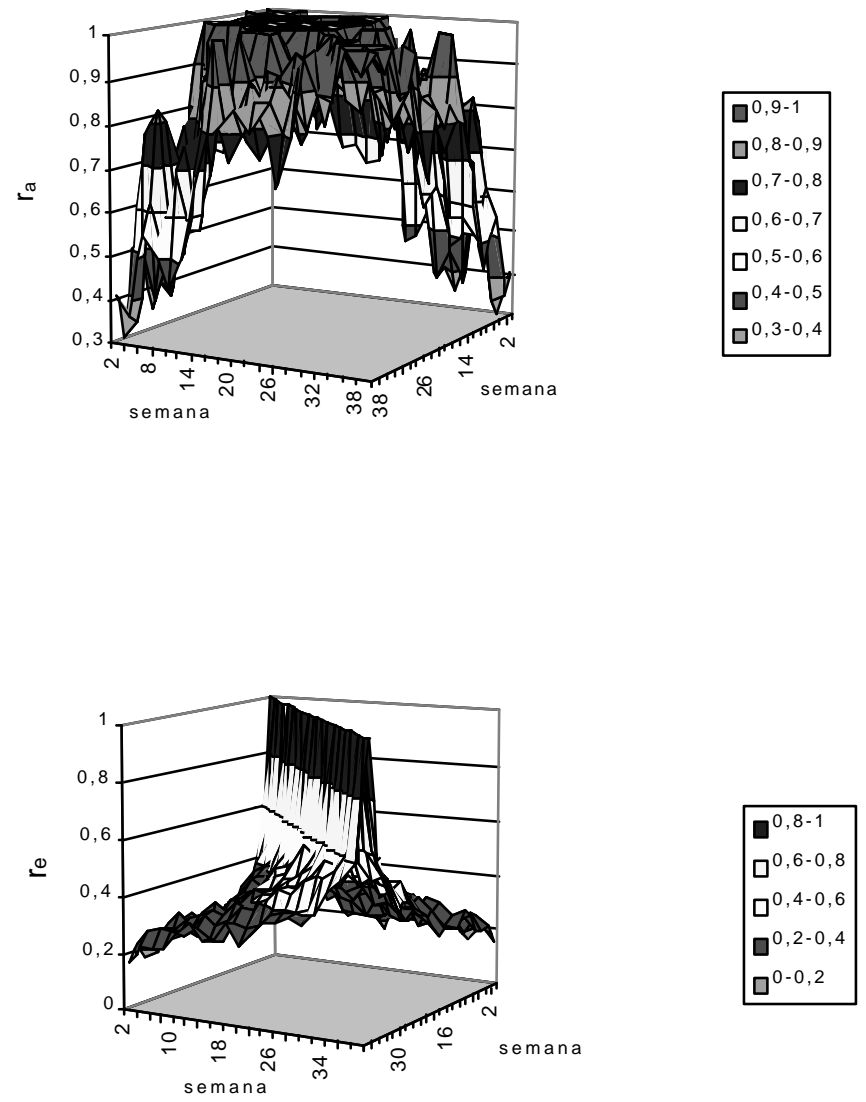

Figura 3 - Correlações fenotípicas (acima), genéticas (meio) e ambientais (abaixo) estimadas entre as produções de leite no dia do controle.

Figure 3 - Phenotypic (up), genetics (mid) and residual (down) correlation estimated among the test day milk yield. 
pequenas, por exemplo entre a 4 e a $30^{\mathrm{a}}$ semanas, implicando em ganhos menores em determinadas fases da lactação, se a seleção tivesse por critério o início ou o final da lactação. Tais resultados indicam que as produções de leite em cada controle não podem ser consideradas por intermédio de um modelo de repetibilidade.

Valores de correlações estimados por Gadini (1997), para vacas Holandesas, apresentaram superfícies mais suaves, com tendências esperadas e sem grandes oscilações. A autora estimou $r_{a}$ altas entre controles consecutivos, variando de 0,95 a 1,00. Em geral, as $r_{a}$ entre as produções pertencentes a controles do meio da lactação foram maiores. Meyer et al. (1989) estimaram $r_{a}$ da ordem de 0,76 a 0,95 entre testes consecutivos. Swalve (1994) obteve altas $r_{a}$ entre as produções de controles consecutivos, variando entre 0,92 e 0,99 , sendo que todas as $r_{a}$ entre os controles mensais foram superiores a 0,70 . Olori (1997), usando controles leiteiros semanais, estimou valores que variaram entre 0,74 (entre as semanas 4 e 40) e 1,00, sendo que controles consecutivos apresentaram sempre valores maiores que 0,90 . Nesse mesmo estudo, o autor observou que os controles adjacentes do meio e do final da lactação apresentaram maiores $r_{a}$ e $r_{p}$ que os controles do início.

A produção total acumulada (P305) apresentou média observada de $1484,27 \mathrm{~kg}$, com desvio-padrão de $343,17 \mathrm{~kg}, \mathrm{CV}$ de $23,12 \%$ e valores de 868,60 a $2796,60 \mathrm{~kg}$.

As estimativas para $\sigma_{a}^{2}, \sigma_{e}^{2}$ e $h^{2}$ foram de $24.944,05 \mathrm{~kg}^{2}, 68.403,46 \mathrm{~kg}^{2}$ e 0,27 , respectivamente, quandoprovenientes de análises uni ou bicaracterísticas. Os valores para a $h^{2}$ estão próximos aos estimados por Albuquerque et al. (1998) e Pelicione (2000), para lactações desse mesmo rebanho, que foram em torno de 0,20 . Vale ressaltar que em ambos os estudos as estimativas de $\mathrm{h}^{2}$ para a $\mathrm{P} 305$ usaram medidas repetidas, justificando assim a maior hi estimada no presente estudo, apenas com primeiras lactações.

Tabela 5 - Estimativas de herdabilidades $\left(h^{2}{ }_{\text {semana }}\right)$ e correlações $\left(r_{a}, r_{e}\right.$ er $\left.r_{p}\right)$ entre as produções dos controles e a produção total de leite (P305); resposta correlacionada (RC) para P305 realizando-se seleção direta para as produções em cada controle e eficiência relativa de seleção (ER\%); resposta correlacionada (RC-cont) para cada controle, selecionandose para P305 e ganho genético (DG) devido a seleção direta para cada controle

Tabela 5 - Estimates of heritabilities $\left(h^{2}{ }_{\text {week }}\right)$ and correlation $\left(r_{a^{\prime}} r_{e}\right.$ e $\left.r_{p}\right)$ among test day milk yields and the total milk yield (M305); correlated response (CR) and relative efficiency of selection (RE\%) to $M 305$ by direct selection to test day milk yields; correlated response (CR-cont) to each test day by selecting for M305 and genetic gain (DG) to direct selection for each control

\begin{tabular}{lcccccccc}
\hline Semana de lactação & $\mathrm{r}_{\mathrm{a}}$ & $\mathrm{r}_{\mathrm{e}}$ & $\mathrm{r}_{\mathrm{p}}$ & $\mathrm{h}_{\text {semana }}{ }_{\text {Week }}$ & $\mathrm{RC}$ & $\mathrm{ER}(\%)^{1}$ & RC-cont & $\Delta \mathrm{G}^{2}$ \\
\hline 2 & & & & & & & & $\mathbf{0 , 4 6}$ \\
4 & 0,67 & 0,46 & 0,51 & 0,22 & 49,86 & 60,44 & $\mathbf{0 , 3 4}$ & 0,27 \\
6 & 0,75 & 0,53 & 0,57 & 0,14 & 46,09 & 55,87 & 0,28 & 0,30 \\
8 & 0,92 & 0,55 & 0,62 & 0,17 & 58,39 & 70,78 & 0,34 & 0,21 \\
10 & 0,86 & 0,56 & 0,60 & 0,13 & 49,20 & 59,64 & 0,26 & 0,18 \\
12 & 0,93 & 0,62 & 0,66 & 0,12 & 55,21 & 66,93 & 0,26 & 0,13 \\
14 & 0,92 & 0,62 & 0,65 & 0,09 & 43,79 & 53,08 & 0,21 & 0,16 \\
16 & 1,00 & 0,61 & 0,67 & 0,12 & 52,62 & 63,79 & 0,24 & 0,16 \\
18 & 1,00 & 0,61 & 0,66 & 0,12 & 59,37 & 71,97 & 0,24 & 0,16 \\
20 & 1,00 & 0,61 & 0,67 & 0,13 & 61,45 & 74,49 & 0,24 & 0,16 \\
22 & 0,99 & 0,65 & 0,70 & 0,13 & 60,84 & 73,75 & 0,23 & 0,19 \\
24 & 1,00 & 0,63 & 0,70 & 0,15 & 67,32 & $\mathbf{8 1 , 6 1}$ & 0,25 & 0,21 \\
26 & 0,95 & 0,65 & 0,71 & 0,17 & 58,38 & 70,77 & 0,25 & 0,16 \\
28 & 0,86 & 0,67 & 0,69 & 0,13 & 51,06 & 61,90 & 0,20 & 0,24 \\
30 & 0,94 & 0,66 & 0,71 & 0,16 & 71,53 & $\mathbf{8 6 , 7 1}$ & 0,24 & 0,19 \\
32 & 0,83 & 0,71 & 0,73 & 0,15 & 51,00 & 61,83 & 0,21 & 0,23 \\
34 & 0,85 & 0,66 & 0,70 & 0,19 & 63,26 & 76,69 & 0,24 & 0,15 \\
36 & 0,93 & 0,70 & 0,73 & 0,12 & 59,02 & 71,55 & 0,22 & 0,21 \\
38 & 0,92 & 0,68 & 0,73 & 0,17 & 54,62 & 66,21 & 0,25 & 0,19 \\
40 & 0,88 & 0,67 & 0,70 & 0,15 & 54,08 & 65,56 & 0,22 & $\mathbf{0 , 3 9}$ \\
42 & 0,86 & 0,59 & 0,67 & 0,31 & 68,23 & $\mathbf{8 2 , 7 1}$ & $\mathbf{0 , 3 2}$ & $\mathbf{0 , 3 2}$ \\
P305 & 0,70 & 0,58 & 0,62 & 0,27 & 61,84 & 74,97 & $\mathbf{0 , 2 3}$ & $\mathbf{8 2 , 4 9}$ \\
\hline
\end{tabular}

1. $E R=(R C / 82,49) 100 ; 2$. resposta esperada devido à seleção direta; 3 . média das análises bicaracterísticas.

1. $R E=(C R / 82,49) 100 ; 2$. response obtained by direct selection; 3. average of bitrait analysis. 
As $\mathrm{h}^{2}$ estimadas para os controles individuais em análises conjuntas com a P305 (Tabela 5) foram iguais às estimativas provenientes das análises uni e bicaracterísticas até a $14^{a}$ semana, com pequenas diferenças nos demais controles. Essa diferença foi maior apenas para a $28^{a}, 40^{a}$ e $42^{a}$ semanas e no final da lactação foi mais herdável para as três análises (Figura 4). Nas discussões a seguir e nos cálculos das repostas correlacionadas (RC e RC-cont), ganho genético $(\Delta \mathrm{G})$ e eficiência relativa (ER), os valores de $h^{2}$ baseiam-se nos apresentados na Tabela 3.

Uma vez que há interesse em verificar a possibilidade de adotar controles individuais como critério de seleção, comparando-se as $\mathrm{h}^{2}$ estimadas para estes controles individuais com a estimada para P305 $(0,27)$, foram observados valores próximos nas $2^{\mathrm{a}}, 28^{\mathrm{a}}, 32^{\mathrm{a}}, 40^{\mathrm{a}}$ e $42^{\mathrm{a}}$ semanas, sendo que para a $42^{\mathrm{a}}$ semana a $\mathrm{h}^{2}$ foi superior, de 0,32 .

As $r_{a}$ entre a P305 e os controles individuais foram todas positivas e elevadas, variando de 0,67 a 1,00 . Os maiores valores ocorreram entre a $10^{\mathrm{a}}$ até a $24^{a}$ semana de lactação e as menores $r_{a}$, apesar de elevadas, ocorreram na 2 e na $42^{\mathrm{a}}$ semana. As $\mathrm{r}_{\mathrm{p}}$ foram positivas e menores que as $r_{a}$ e os valores estimados para $r_{e}$ e $r_{p}$ foram próximos.

É interessante notar que, apesar da $\mathrm{h}^{2}$ estimada para a $42^{\mathrm{a}}$ semana de lactação ser mais elevada, sua $\mathrm{r}_{\mathrm{a}}$ com a P305 foi de 0,70 . Por outro lado, os controles com as menores estimativas de $\mathrm{h}^{2}$, ocorridas no meio da lactação, apresentaram as maiores $r_{a}$ com a P305. A seleção direta para as produções parciais traria ganhos também para a P305, principalmente se baseada nos controles 22, 28, e 40 (Tabela 5). Entretanto, considerando-se que a intensidade de seleção fosse constante, maiores ganhos seriam alcançados para $\mathrm{P} 305$ por intermédio de seleção direta. É importante ressaltar que poderia haver maior ganho genético para P305 por meio de seleção indireta, se a intensidade de seleção fosse maior, uma vez que maior número de informações de touros jovens estaria disponível usando-se os controles individuais. Além disso, a possibilidade de se realizar a seleção mais cedo proporcionaria menores intervalos de geração, implicando, assim, em maiores ganhos. A seleção baseada na P305, o que já vem sendo realizada pela propriedade, implicaria em maior resposta correlacionada para a produção de leite em todas as fases da lactação, exceto na $2^{\mathrm{a}}, 40^{\mathrm{a}}$ e $42^{\mathrm{a}}$ semanas.

Para gado Holandês os estudos têm relatado que as maiores $h^{2}$ têm sido estimadas para o $4^{\circ}$ ou $5^{\circ}$ mês de lactação, sendo que alguns estudos têm obtido tais

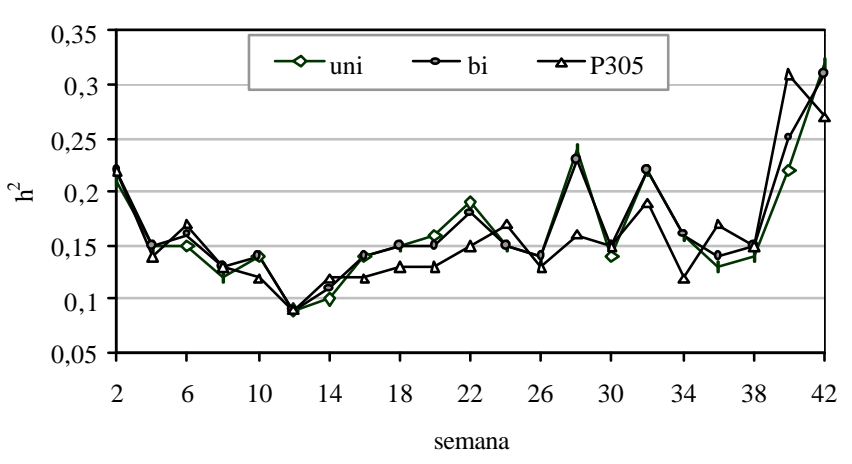

Figura 4 - Estimativas de $h^{2}$ para gc2, de acordo com as análisesunicaracterísticas (uni) ebicaracterísticas entre controles (bi) e entre controles e a P305.

Figure 4 - Estimates of $h^{2}$ to gc2, by unitrai (uni) and bitrai analysis among test days (bi) and test days and M305.

valores mais para o final da lactação, do 6 ao $10^{\circ}$ controles (Pander et al., 1992; Reents et al., 1994; Rekaya et al., 1995; Olori, 1997). Os valores de $h^{2}$ têm se mostrado menores ou próximos aos estimados para a P305, com $r_{a}$ altas e positivas, principalmente entre os controles do meio da lactação e as P305. O início e o final têm apresentado as menores $r_{a}$ com a P305. Tais resultados validam os encontrados no presente estudo, cujas $\mathrm{h}^{2}$, em alguns controles, foram bem próximas às relatadas na literatura, com elevadas $r_{a}$.

Na definição dos critérios de seleção, as estimativas de correlações genéticas entre as produções de leite dos controles e entre estas com a P305 ajudam a visualizar prováveis perdas ou ganhos em fases da lactação, dependendo do objetivo de seleção desse rebanho. A seleção direta usando apenas um controle leiteiro como critério de seleção poderia trazer perdas para a P305, não possibilitando a estimação de componentes da curva de lactação, como, por exemplo, a persistência. A definição de índices contendo vários controles e componentes da curva de lactação poderia contornar estes problemas. Meyer et al. (1989) propuseram índices canônicos não apenas como uma alternativa para minimizar os requerimentos computacionais dessas análises multivariadas, mas para gerar variáveis canônicas que fossem genética e fenotipicamente independentes entre si. As variáveis canônicas analisadas que tivessem os maiores autovalores associados a elas seriam responsáveis então pelas maiores variações genéticas correspondentes. No estudo desses autores, um índice canônico contendo os três ou quatro primeiros con- 
troles apresentou maiores $\mathrm{h}^{2}$ associadas, da ordem de 0,3 a 0,4 , e, quando cinco ou mais controles eram considerados no índice, a $\mathrm{r}_{\mathrm{a}}$ com a P305 foi muito próxima de um.

\section{Conclusões}

Dadas as correlações genéticas entre os diferentes controles quinzenais estimadas no presente estudo, que foram altas, porém diferentes da unidade, a adoção de um modelo de repetibilidade para a avaliação genética de bovinos leiteiros, possivelmente, não seria adequada.

A utilização dos controles do final da lactação como critérios de seleção não justificaria a utilização de test day model para futuras avaliações genéticas. Os resultados obtidos indicam que a seleção de animais com lactações em andamento, baseada na produção de leite pertencente a controles do início ou meio da lactação, seria menos eficiente, já que o final da lactação se mostrou mais herdável.

A P305 é o melhor critério, uma vez que a seleção direta para essa característica implicaria em maiores ganhos genéticos para as produções da maioria dos controles da lactação, além de maior ganho para a própria $\mathrm{P} 305$.

\section{Literatura Citada}

ALBUQUERQUE, L.G.; FRIES, L.A.; QUEIROZ, S.A. Correlação genética entre produção de leite e crescimento prédesmame em bovinos da raça Caracu. In: REUNIÃO ANUAL DA SOCIEDADE BRASILEIRA DE ZOOTECNIA, 35., 1998, Botucatu. Anais... Botucatu: Sociedade Brasileira de Zootecnia, 1998. CD ROM.

BIANCHINI SOBRINHO, E. Estudo da curva de lactação de vacas da raça Gir. Ribeirão Preto: Universidade de São Paulo, 1984. 88p. Tese (Doutorado em Genética) - Universidade de São Paulo, 1984.

BOLDMAN, K.G.; KRIESE, L.A.; Van VLECK, L.D. et al. A manual for use of MTDFREML. USDA-ARS: Clay Center, Nebraska, USA. 1993. 120p.

GADINI, C.H. Genetic evaluation of test day production traits and somatic cell score. Lincoln: University of Nebraska, 1997.91p. Thesis (PhD) - University of Nebraska, Nebraska, 1997.

KETTUNEN, A.; MANTYSAARI, E. A.; STRANDÉN, I. et al. Estimation of genetic parameters for first lactation test day milk production using Random Regression Models. In: WORLD CONGRESS OF GENETICS APPLIED TO LIVESTOCK PRODUCTION, 6., 1998, Armidale, Australia. Proceedings... Armidale: University of New England, 1998. p.307-310.
MACHADO, S.G.; FREITAS, M.A.R.; GADINI, C.H. Genetic parameters of test-day milk yield of Hostein cows. In: WORLD CONGRESS OF GENETICS APPLIED TO LIVESTOCK PRODUCTION, 6., 1998, Armidale, Australia. Proceedings... Armidale: University of New England, 1998. p.427-430.

MEYER, K., GRASER, H.U., HAMMOND, K. Estimates of genetic parameters for first lactation test day production of Australian Black and White cows. Livestock Production Science, v.21, p.177-199, 1989.

OLORI, V.E. Utilization of daily milk records in genetic evaluation of dairy cattle. Edinburgh: University of Edinburg, 1997. Thesis (PhD) - University of Edinburg, 1997.

PANDER, B.L.; HILL, W.G.; THOMPSON, R. Genetic parameters of test day records of British Holstein-Friesian heifers. Animal Production, v.55, p.11-21, 1992.

PELICIONE, L.C. Polinômios segmentados no ajuste de idade e efeitos da linhagem citoplasmática para características de crescimento e produção de leite em bovinos da raça Caracu. Jaboticabal: Universidade Estadual Paulista, 2000. 112p. Dissertação (Mestrado em Zootecnia) - Universidade Estadual Paulista, 2000.

PTAK, E.; SCHAEFFER, L.R. Use of test day yields for genetic evaluation of dairy sires and cows. Livestock Production Science, v.34, p.23-34, 1993.

REKAYA, R.F; BÉJAR, F.; CARABAÑO, M.J. et al. Genetic parameters for test day measurements in Spanish Holstein Friesian. In: INTERBULL ANNUAL MEETING, 1995, Prague. Proceedings... Prague, 1995. p.1-8.

REENTS, R.; DEKKERS, J.C.M.; SCHAEFFER, L.R. Genetic parameters of test day somatic cell counts and production traits. In: WORLD CONGRESS OF GENETICS APPLIED TO LIVESTOCK PRODUCTION, 5., 1994, Guelph. Proceedings... Guelph: University of Guelph, 1994. p. 120-122.

SWALVE, H.H. Genetic relationship between dairy lactation persistency and yield. Journal of Animal Breeding and Genetics, v.112, p.303-311, 1995a.

SWALVE, H.H. The effect of Test day models on the estimation of genetic parameters and breeding values for dairy yield traits. Journal of Dairy Science, v.78, p.929-938, 1995b.

SWALVE, H.H. Use of test day records for genetic evaluation. In: WORLD CONGRESS OF GENETICS APPLIED TO LIVESTOCK PRODUCTION, 6., 1998, Armidale, Australia. Proceedings... Armidale: University of New England, 1998. p.295-302.

WILMINK, J.B.M. Adjustment of test-day milk, fat and protein yield for age, season and stage of lactation. Livestock Production Science, v.16, p.335-348, 1987. 